\title{
DINAMISMO ECONÓMICO DEL TURISMO A ESCALA LOCAL. UNA APROXIMACIÓN A TRAVÉS DEL ANÁLISIS DE CLASES LATENTES
}

\author{
Juan Antonio Parrilla-González \\ Juan Ignacio Pulido-Fernández. \\ Universidad de Jaén
}

\section{RESUMEN}

Hasta hace escasos años, el destino turístico era relegado por la literatura científica al papel de mero contenedor de la actividad turística. Sin embargo, durante la última década, se empieza a asumir la importancia del destino en la toma de decisiones del turista y en la satisfacción de sus necesidades y expectativas, lo que conlleva la necesidad de una gestión activa de este tipo de territorios. Por otro lado, el continuo crecimiento de la demanda turística anima a un número cada vez mayor de territorios a poner en valor sus recursos y atractivos para posicionarse y conseguir o aumentar cuota de mercado en el negocio turístico. Ello genera una creciente competencia entre destinos que, de nuevo, conlleva la necesidad de una gestión activa de los destinos turísticos. En este artículo se demuestra la importancia de analizar el dinamismo económico del turismo, como herramienta para la segmentación de destinos, lo que facilitará a los policymakers y gestores de destinos la adopción de medidas de política turística y de gestión diferenciadas según el nivel más o menos avanzado de cada territorio.

Palabras clave: destino turístico, dinamismo económico del turismo, gestión de destinos, análisis de clases latentes, Andalucía (España).

Recibido: 8 de octubre de 2015

Devuelto para su revisión: 4 de mayo de 2016

Aceptado: 15 de julio de 2016

Área de Economía Aplicada. Universidad de Jaén. Campus Las Lagunilla. 23071 JAÉN (España). E-mail: japg0003@red.ujaen.es,jipulido@ujaen.es 
Economic dynamism of tourism on local scale. An approach through Latent Classes Analysis

\begin{abstract}
Until a few years ago, the destination was relegated from the scientific literature to the role of mere container of tourism. However, during the last decade, it begins to assume the importance of the destination in the decision-making tourist and meeting their needs and expectations, leading to the need for active management of such territories. Furthermore, the continued growth of tourism demand encourages a growing number of territories to value their resources and attractive position and get or increase market share in the tourism business. This generates increased competition between destinations which, again, involves the need for active management of tourist destinations. In this article is evidenced the importance of analyzing the economic dynamism of tourism as a tool for segmenting destinations, which make it easier for policymakers and managers destinations to adopt measures of tourism policy and management differentiated according to the level more or less advanced in each territory.
\end{abstract}

Keywords: tourism destination, economic dynamism of tourism, destinations management, latent class analysis, Andalusia (Spain).

\title{
1. INTRODUCCIÓN
}

En los últimos años, el destino turístico ha despertado interés en los estudios relacionados con la planificación económica del turismo, especialmente desde el punto de vista mesoeconómico. Candela y Figini (2012) destacan la evolución del interés por el destino turístico desde una perspectiva científica, puesto que, hasta muy recientemente, todos los estudios se centraban en la dimensión microeconómica o en los agregados macroeconómicos, relegando el análisis del destino al de mero contenedor de productos y/o servicios.

Autores como Saraniemi y Kylanen (2011) o Pearce (2014) justifican que el de destino turístico es un concepto clave, que debe ser entendido por todos los agentes implicados en la planificación y la gestión del turismo, puesto que su definición es un elemento crítico por razones prácticas. Es decir, la conceptuación del término destino turístico tendrá repercusión a la hora de plantear y abordar problemas, dará forma al diseño de la investigación y la metodología e influirá en la interpretación de los hallazgos. Además, varios autores apuntan que los destinos constituyen la unidad de análisis apropiada para la investigación del turismo (Blasco et al., 2014).

Pero, como señalan Pulido-Fernández y Pulido-Fernández (2013:179), "un destino no es sólo un territorio en el que existen un conjunto de agentes, más o menos coordinados y organizados, involucrados en la producción y provisión del producto turístico, sino que es lo que realmente se posiciona en el imaginario colectivo de los potenciales clientes como el territorio en el que disfrutar de una experiencia memorable".

En tal contexto, se establece como hipótesis de esta investigación que es posible analizar el dinamismo económico del turismo (en adelante DET) a escala local, mediante un 
conjunto de variables económicas vinculadas con la oferta de los destinos turísticos y, a partir de ahí, segmentar estos destinos turísticos en función de su mayor o menor DET.

Esta segmentación, que permite conocer el carácter dinámico de cualquier destino turístico, será muy útil en futuras investigaciones para averiguar en qué medida el hecho de que un municipio sea más dinámico desde el punto de vista turístico favorece los procesos de desarrollo local, lo que vendría a demostrar que el turismo es una importante herramienta de desarrollo.

El estudio que se propone se focaliza en Andalucía, una de las regiones españolas de mayor peso turístico. Para verificar la hipótesis, se trabajará con un conjunto de variables que permiten seleccionar y segmentar los municipios de Andalucía en grupos, atendiendo a su DET, durante un período de 15 años. De esta manera, se obtendrá una clasificación de los municipios andaluces según su mayor o menor DET.

\section{MARCO TEÓRICO}

Se realiza en este apartado una aproximación al concepto de destino turístico a través de los diferentes autores que han centrado su interés en la importancia del destino como elemento aglutinador de la oferta turística y territorio en el que el turista consume y disfruta de la experiencia turística.

Tomando como base los estudios de Pearce (2014) y Candela y Figini (2012), a continuación se desgrana todo lo relacionado con el término destino turístico, desde una perspectiva mesoeconómica.

Además, se ofrece una visión dinámica del destino turístico, en la que se pretende conocer cómo interactúa el conjunto de elementos que conforma un destino turístico, creando valor en su actividad, y entre los stakeholders que rodean el entorno local, contribuyendo así al efecto multiplicador del turismo a escala local, lo que, desde el punto de vista de la economía del turismo, supone un aspecto clave en la implantación del turismo a escala local.

\subsection{Visión mesoeconómica del destino turístico}

La evolución de las investigaciones en turismo ha reconocido al destino turístico como un concepto central en el campo de estudio relacionado con la economía del turismo (Candela y Figini, 2012). De hecho, Pearce (2014) plantea la necesidad de revisar los conceptos previos que conducen al de destino turístico y sus variaciones. Para ello, es necesario hablar del marco conceptual previo relacionado con los distritos industriales, cuya referencia más importante es Becattini (1990), y los clusters, donde los estudios de Porter (1998) son los más relevantes.

Además, hay que considerar el análisis de redes (Scott, Cooper y Baggio, 2008a; Pearce, 2012), los planteamientos de la Teoría General de Sistemas (Weaver y Oppermann, 2000) y construcciones sociales (Iwashita, 2003; Saarinen, 2004; Ringer, 1998), para completar la visión mesoeconómica de destino turístico, su relación con el territorio y con el desarrollo de éste. Por tanto, es fundamental conocer todos estos aspectos teóricos y su relación, la cual desemboca en dimensiones relacionadas con la concepción geográfica, el dinamismo de estos destinos y su modo de organización y producción para generar valor (Pearce, 2014). 
Respecto a la comparación entre distrito industrial y destino turístico, destacan estudios de investigadores europeos (Aurioles, Fernández y Manzanera, 2008; Capone, 2006; Gaido, 2002; Hjalager, 2000; Maulet, 2006; Petric y Mrnjavac, 2003; Sainaghi, 2006). Por su parte, Becattini (1990: 38) definió el distrito industrial como "una entidad socio-territorial caracterizada por la presencia activa de una comunidad de personas y una población de empresas enraizadas de forma natural en un territorio". Este autor pone especial énfasis en la manera en que se aprecia una actividad especializada en un entorno local, generando valores y reglas que dependen de la confianza y las relaciones personales.

Becattini también resalta la naturaleza dinámica del distrito industrial, como un "sistema adaptativo" que evoluciona en respuesta a las necesidades del mercado y las condiciones cambiantes del entorno que lo rodea, donde los valores son transmitidos de una generación a otra, los recursos humanos están en un proceso de constante recolocación y la tecnología está en continuo avance, adaptándose a un proceso social.

Algunos investigadores sugieren similitudes entre los conceptos de distrito industrial y destino turístico (Pearce, 2014), pudiendo considerarse el destino turístico, desde la perspectiva del concepto de distrito industrial, como un "modo de organización y producción" en el que se persigue, desde una perspectiva local, generar valor a las actividades del distrito, a través de la interconexión e interdependencia de empresas, cooperación y competitividad y confianza entre actores socioeconómicos (Hjalaguer, 2000). Petric y Mrnjavac (2003) comparan los destinos turísticos con los distritos industriales y los modelos de desarrollo sostenible con base en la organización funcional y espacial, el producto y el proceso de producción, las empresas, la conciencia sociocultural y la conexión con los stakeholders para generar valor.

Un segundo concepto relacionado con el carácter mesoeconómico de los destinos turísticos es el de cluster. Se trata de un modelo organizativo aplicado y popularizado por Porter (1998), quien explicó el diferente rol de las localizaciones creado por la ventaja competitiva en una economía global, a través de los beneficios de proximidad e interconexión de las empresas establecidas en dicha localización.

De acuerdo con Porter (1998: 78), los clusters son "concentraciones geográficas de empresas e instituciones interconectadas en un campo específico". En este sentido, el rol de la localización no es más que la visión tradicional dada en lo relacionado con la ventaja comparativa, pues, según esta teoría, es el beneficio de la localización lo que hace al territorio competitivo.

Para Pearce (2014), la productividad de los actores del territorio incrementa al formar parte de un cluster, ya que los clusters son capaces de mejorar el acceso al empleo y a otras ramas de actividad, especializarse y compartir actividad en una cadena de valor y trabajar con información también especializada y con instituciones en las que se provee de motivación y confianza.

Uno de los aspectos más importantes de los clusters es el denominado "beneficio de la complementariedad", es decir el derivado de la generación de sinergias, esto es, que el todo tiene un impacto y una escala mayor que la suma de sus partes por separado. Porter usa el turismo para ilustrar este último aspecto.

Por otra parte, adoptando la perspectiva del análisis de redes en turismo, los investigadores reconocen la amplitud de stakeholders involucrados en un destino turístico y la 
importancia de entender las relaciones entre ellos, especialmente desde la perspectiva de la experiencia del turista (Camprubi, Guia y Comas, 2008; Denicolai, Cioccarelli y Zucchella, 2010; Pavlovich, 2003; Scott, Cooper y Baggio, 2008b). Merinero-Rodríguez y Pulido-Fernández (2009), en un estudio sobre análisis de redes en la región de Andalucía (España), revelan la existencia de una relación positiva entre la estructura de las redes y los niveles de desarrollo turístico a escala local.

El término sistema se utiliza también para delimitar desde un punto de vista conceptual una aproximación teórica hacia lo que puede ser el destino turístico. Weaver y Oppermann (2000: 23), conceptualizan el término sistema como "un grupo de elementos interrelacionados, interdependientes e interactivos que juntos forman una estructura funcional". Siguiendo con esta definición, Capone (2006: 10) resalta, en relación con el turismo, el concepto de sistema turístico local, que, en términos de sistema, "las actividades integradas y enraizadas en un territorios requieren de la coordinación y el compromiso de todos los stakeholders", así, las características locales, el rango de productos turísticos y un sistema local abierto a las relaciones con el mundo externo, conforman esta definición (Pearce, 2014).

Para terminar esta aproximación conceptual de lo que es un destino turístico, es necesario hablar del social-construccionismo, ya que diversos investigadores han aportado un carácter social a este concepto, entendiéndolo como social construccionismo. Iwashita (2003: 331) concibe el social construccionsimo como "el principio por el cual los fenómenos sociales y culturales se convierten en construcciones sociales producidas y reproducidas históricamente y culturalmente, generando interacciones de personas y actividades humanas en una sociedad".

Autores como Ringer (1998:2) conceptualizan los destinos turísticos entendiéndolos como destinos comunitarios basados en construcciones sociales, en los que "grupos de personas pertenecientes a lugares con experiencias vividas reflejan un paisaje cultural determinado y las economías locales exhiben cada vez más la afluencia de nuevas ideas y cambios en los patrones de interpretación y comunicación social asociados con el progreso del turismo" y, en definitiva, y como conclusión, resalta que "el turismo es un proceso cultural tanto como una forma de desarrollo económico".

Para concluir, señalar que Candela y Figini (2012) han identificado dos teoremas específicos que permiten interpretar el destino turístico como un tipo particular de distrito económico, que comparte algunas de las características de la zona industrial y algunas otras del distrito cultural. Se trata del love of variety theorem y el coordination theorem. Estos dos teoremas identificados por Candela y Figini (2012) hacen referencia a dos aspectos clave que, como se verá seguidamente, están íntimamente relacionados con el concepto de DET que se estudia en este artículo.

\subsection{Dimensión dinámica de los destinos turísticos}

Desde los años ochenta del siglo XX, ha habido un incremento de los destinos turísti$\cos \mathrm{y}$, consecuentemente, un aumento de la competencia entre los mismos. Para mejorar su desempeño competitivo, algunos destinos más consolidados apuestan por un modelo turístico diverso, de calidad y sostenible. Además, con el objetivo de la competitividad, 
es importante no sólo la puesta en valor sostenible de los recursos de dicho territorio, sino que cada vez cobra mayor importancia la habilidad que tienen los agentes del destino para añadir valor a los recursos existentes y obtener de ellos la mayor rentabilidad posible; lo que influirá, a su vez, en la calidad de vida de la población local.

Así, se puede realizar una aproximación a la naturaleza dinámica de los destinos turísticos, más enfocada en el desarrollo estructural de los mismos. Para Pearce (2014), el destino no es estable y sólo es estable en la estructura socio-espacial, mientras que aspectos como la cultura del destino están en continua transformación, en la cual emergen cambios, y desaparecen y reaparecen diferentes formas relacionadas con elementos culturales del entorno.

Con todo lo anterior, el dinamismo turístico (en adelante, DT) se puede definir y explicar a partir de la adaptación del "modelo del pentágono" de Coccossis, Janssen, Kiers y Nijkamp (1991) al turismo (Figura 1).

\section{Figura 1 \\ MODELO DEL PENTÁGONO DEL DT}

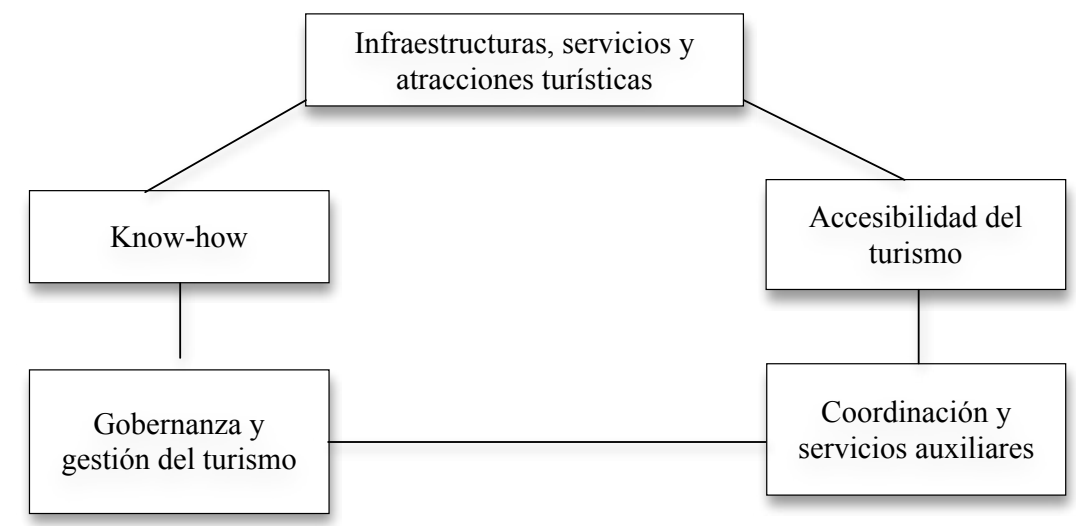

Fuente: elaboración propia a partir de Coccossis, Janssen, Kiers y Nijkamp (1991) y Candela y Figini (2012).

A partir de los planteamientos de Candela y Figini (2012), se pueden establecer cinco grandes ámbitos que explican el DT. Como ya se ha destacado, el destino turístico es una amalgama, una mezcla en la que pueden ser identificados los siguientes componentes:

- Las infraestructuras, servicios y atracciones turísticas que conforman todas aquellas estructuras e iniciativas que sirven de base a los procesos de cambio estructural en la actividad turística. Candela y Figini (2012) los definen como aquellos que, de manera artificial, natural o cultural, interrelacionan y generan recursos que satisfacen el principal propósito del viaje y los cuales se encuentran presentes en todas las facetas y puntos estratégicos del destino turístico.

- La accesibilidad del turismo, en relación a un destino turístico es considerada como la accesibilidad desde la eficiencia del sistema de movilidad local y hacia el destino. 
- La coordinación y los servicios auxiliares son fundamentales, ya que, durante la estancia, el turista necesita alojamiento, transporte, esparcimiento y actividades culturales, alimentos y bebidas, etc., y todos estos bienes y servicios tienen que ser suministrados de manera coherente y organizada. Esta característica también mide el grado de relación de los establecimientos turísticos del destino o la capacidad de innovación turística del mismo (Hjalager, 2000; Cunha Barbosa y Aricó Zamboni, 2001; Hawkins, 2004; Varisco, 2004).

- El know-how turístico, la gobernanza y la gestión están formados por aquellas características intrínsecas de la actividad turística en el destino y que tienen un carácter tácito. Dentro de este pilar se puede incluir la relación del turismo con la cualificación de los recursos humanos, relacionando especialmente su grado de formación en la actividad turística, la gestión de los actores y políticas participativas, la capacidad emprendedora e innovadora existente, el conocimiento acumulado de las rutinas organizativas de las empresas o el nivel cultural de la población residente y de los turistas (Bodega, Ciocarelli y Denicolai, 2004; Timur y Getz, 2008; Fernandes y Thomas, 2012).

Además del análisis anterior, según Sáez (2008), hay que tener en cuenta que el turismo, como herramienta de desarrollo, puede no suponer el éxito de un territorio de cara a afrontar los desafíos de crecimiento, transformación y bienestar, puesto que factores como la diversidad y madurez de la economía local, la disponibilidad de recursos financieros para invertir o el tipo y escala del desarrollo turístico pueden limitar el desarrollo de una comunidad determinada y, consecuentemente, los beneficios económicos que genera el turismo.

Para finalizar, hay que resaltar que la aproximación conceptual realizada en este apartado sobre DT está hecha desde una perspectiva de oferta. Sin embargo, es evidente que un destino turístico, más allá de ser un territorio cuya gestión se orienta hacia los ciudadanos residentes, tiene su razón de ser en la medida en que responde, además, a las necesidades y expectativas de sus visitantes. Por tanto, la adaptación realizada en este apartado del marco analítico tradicional para el análisis del dinamismo territorial debería completarse en un futuro incorporando la perspectiva de demanda, lo que exige contar con información de demanda a escala local, que hoy día no está disponible.

Por otra parte, en este trabajo, el análisis de la importancia de la interacción entre el conjunto de los elementos que conforman el destino turístico se ha realizado exclusivamente desde la perspectiva de la economía del turismo, que es el ámbito de especialización de sus autores. No obstante, es justo reconocer que, si bien el análisis sirve a los objetivos de este artículo, esta percepción es limitada, ya que han sido muchos los campos de conocimiento de las Ciencias Sociales que han contribuido a este avance conceptual.

\section{MARCO METODOLÓGICO}

Una cuestión a tener en cuenta en la investigación que se muestra en este artículo es poner en evidencia la dificultad para disponer de indicadores desagregados que permitan realizar un análisis profundo a nivel local, en el que se pueda ofrecer una perspectiva 
completa del DT. Como consecuencia, esta dificultad nos obliga a utilizar un concepto más restringido que el que se ha definido en el marco teórico y que analiza desde una perspectiva económica y de la oferta el DT, al que se ha denominado DET. Por tanto, como se recoge en la Figura 2, el DT engloba tanto las variables económicas (de las que actualmente es más fácil conseguir información), como aquellos indicadores que en la actualidad no están desagregados a nivel local, mientras que el DET recoge solo aquellas variables económicas medibles a nivel local: infraestructuras, accesibilidad y coordinación y servicios auxiliares.

\section{Figura 2 \\ RELACIÓN ENTRE LOS CONCEPTOS DINAMISMO TURÍSTICO Y DINAMISMO ECONÓMICO DEL TURISMO}

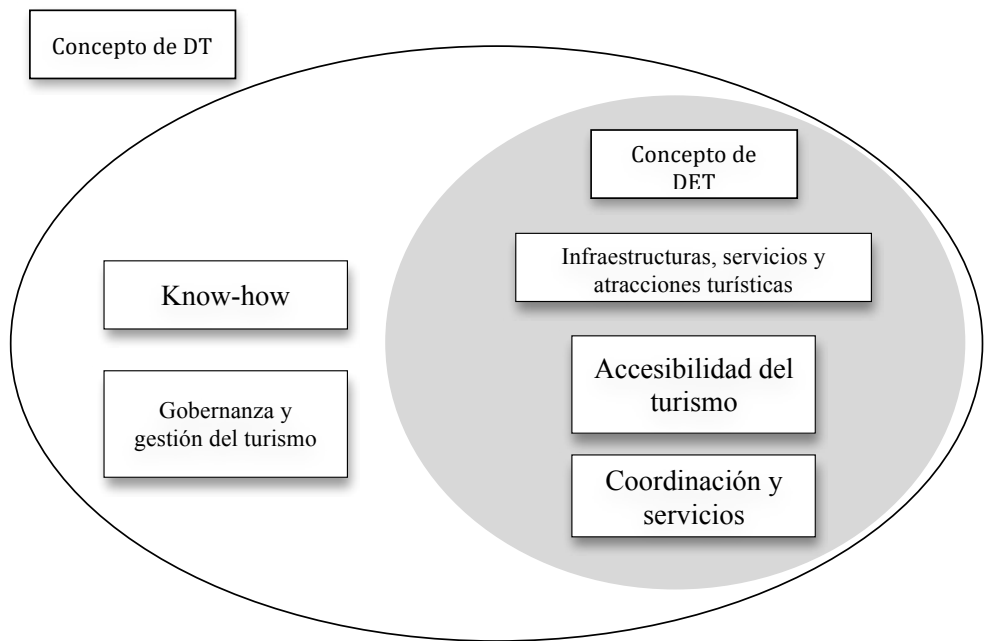

Fuente: elaboración propia.

En consecuencia, en este artículo se va a entender por DET el crecimiento producido en la oferta turística de un destino como consecuencia de la evolución positiva, en un determinado período, de un conjunto de variables de carácter económico que afectan directamente al mismo.

Se expone en este apartado el proceso metodológico seguido para la realización de esta investigación. Como se aprecia en la Figura 3, se ha procedido a clasificar el total de 769 municipios que conforman la región de Andalucía según su DET, en el período 1996-2011, a través del método del análisis de clases latentes, para, finalmente estudiar el comportamiento de los grupos de municipios identificados y plantear un análisis pormenorizado en los municipios que resulten dinámicos.

El DET se ha considerado, por tanto, como una variable latente, es decir, no directamente observable, fruto de la evolución de un conjunto de variables de carácter económico que sí que se pueden medir. 


\section{Figura 3 \\ PROCESO METODOLÓGICO}

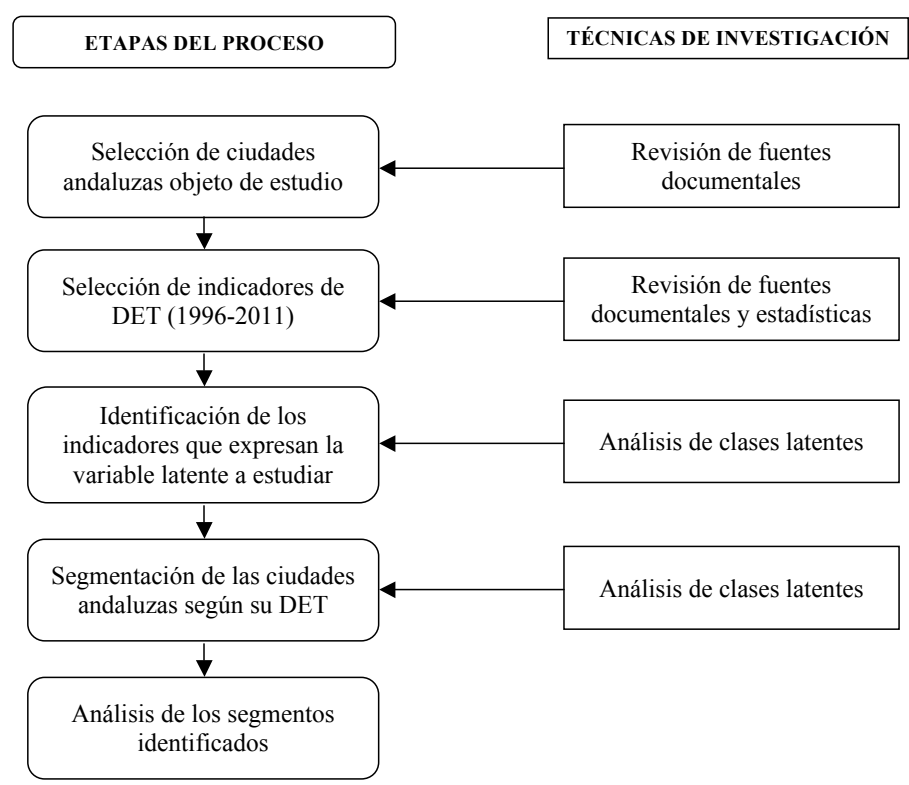

Fuente: elaboración propia.

\subsection{Selección de los datos}

Se dispone de un total de $n=769$ municipios de Andalucía. La elección de esta región para la elaboración de este estudio se justifica por la importancia del turismo en la misma, que generó 22,5 millones de turistas y 16,2 miles de millones de euros en 2013, lo que supuso una contribución a la economía andaluza del 12,3\% del PIB (Consejería de Turismo, Comercio y Deporte, 2013).

Para cada uno de estos municipios, se han observado $p=30$ indicadores estadísticos que se corresponden con características de índole turística en un horizonte temporal lo suficientemente amplio que permita medir de forma significativa la evolución de dichas variables, por lo que se ha utilizado un horizonte temporal comprendido entre los últimos años del siglo XX y los primeros del siglo XXI, es decir, que abarca desde 1996 hasta 2011 (15 años). El año final de referencia es 2011 porque es el último para el que se dispone de datos para alguno de los indicadores utilizados en este estudio.

Una vez establecido dicho período, se han calculado las tasas de variación relativas (TVR) para cada indicador, donde se establece $t_{\text {inicial }} y t_{\text {final }}, \mathrm{y}$ se calcula su correspondiente tasa de variación relativa (TVR), según la fórmula:

$$
T V R^{i}=\frac{X_{t_{\text {final }}}^{i}-X_{t_{\text {inicial }}}^{i}}{X_{t_{\text {inicial }}}^{i}}, \quad i=1, \ldots, 30
$$


Tabla 1

CONJUNTO DE INDICADORES RELACIONADOS CON EL DET

\begin{tabular}{|c|c|}
\hline Indicadores de DET & Fuente \\
\hline Museos & Consejería de Educación, Cultura y Deporte \\
\hline Plazas en hoteles & Consejería de Turismo y Comercio \\
\hline Plazas Hoteles - Apartamento & Consejería de Turismo y Comercio \\
\hline Plazas Hostales y Pensiones & Consejería de Turismo y Comercio \\
\hline Plazas Apartamentos 4 Llaves & Consejería de Turismo y Comercio \\
\hline Plazas Apartamentos 3 Llaves & Consejería de Turismo y Comercio \\
\hline Plazas Apartamentos 2 Llaves & Consejería de Turismo y Comercio \\
\hline Plazas Apartamentos 1 Llave & Consejería de Turismo y Comercio \\
\hline Desempleo en actividad & Observatorio ARGOS - Consejería de Economía, Innovación, Ciencia \\
\hline turística & y Empleo \\
\hline Cines & $\begin{array}{l}\text { AIMC. Asociación para la Investigación de Medios de } \\
\text { Comunicación }\end{array}$ \\
\hline Pantallas de cine & $\begin{array}{l}\text { AIMC. Asociación para la Investigación de Medios de } \\
\text { Comunicación }\end{array}$ \\
\hline Aforo de cines & $\begin{array}{l}\text { AIMC. Asociación para la Investigación de Medios de } \\
\text { Comunicación }\end{array}$ \\
\hline Bancos & Banco de España. Boletín estadístico \\
\hline Cajas de ahorro & Banco de España. Boletín estadístico \\
\hline Cooperativas de crédito & Banco de España. Boletín estadístico \\
\hline Campamentos & Consejería de Turismo y Comercio \\
\hline Hoteles & Consejería de Turismo y Comercio \\
\hline Hoteles - Apartamento & Consejería de Turismo y Comercio \\
\hline Hostales y Pensiones & Consejería de Turismo y Comercio \\
\hline Restaurantes & Consejería de Turismo y Comercio \\
\hline Cafeterías & Consejería de Turismo y Comercio \\
\hline Taxis & Consejería de Obras Públicas y Vivienda \\
\hline Alquiler coches y conductor & Consejería de Obras Públicas y Vivienda \\
\hline Ambulancias & Consejería de Salud \\
\hline Transporte discreccional & Consejería de Obras Públicas y Vivienda \\
\hline Transp. Público + 10 Viajeros & Consejería de Obras Públicas y Vivienda \\
\hline Transp. Público - 10 Viajeros & Consejería de Obras Públicas y Vivienda \\
\hline IAE División 6 & Consejería de Economía, Innovación, Ciencia y Empleo \\
\hline Índice turístico & Anuario Económico de España - La Caixa \\
\hline Oficinas de turismo & Consejería de Turismo y Comercio \\
\hline
\end{tabular}

Fuente: elaboración propia. 
Obviamente, todas las características observadas son de naturaleza cuantitativa, establecidas por los indicadores recogidos en la Tabla 1, por lo que las tasas de variación relativa son también variables cuantitativas. Concretamente, y a diferencia de lo que ocurría con la mayor parte de las características observadas inicialmente, las tasas son variables cuantitativas continuas, y su rango de variación es la totalidad del espacio real. Estas tasas son, además, adimensionales y vienen expresadas en tanto por uno.

Cabe recordar que, para el cálculo del DET, se tendrá en cuenta que hay variables, como el desempleo en la actividad turística, cuyo crecimiento afecta negativamente a la variable latente, por lo que se considerará el correspondiente signo negativo.

\subsection{Análisis de clases latentes}

El análisis de clases latentes (Lazarsfeld y Henry, 1968), también conocido como mezcla de componentes normales o análisis discriminante latente, es una metodología estadística para la clasificación en grupos de objetos similares, en la que ni el número de grupos ni la forma de cada uno de ellos son conocidos a priori. Por forma de un grupo se entiende los parámetros del mismo; esto es, su media, su varianza y sus covarianzas.

Los análisis que incorporan variables latentes tienen, por lo general, una doble finalidad: por un lado, detectar si las relaciones entre las variables manifiestas pueden ser explicadas por una o más variables latentes y, por otro, definir criterios fiables para la clasificación en grupos de los individuos de la muestra. Efectivamente, al igual que la mayoría del resto de técnicas estadísticas multivariantes, los análisis de este tipo persiguen una reducción de la dimensión de los datos y una caracterización de las relaciones entre las variables observadas. Por otra parte, son muy habituales las situaciones en las que es necesario establecer patrones o clases para agrupar individuos según características que no son observables directamente. En este sentido, los análisis de variables latentes constituyen una herramienta muy potente que permite la creación de distintos grupos o clases y la asignación de cada individuo a uno de ellos, en función de los valores que, para ese individuo, toman las variables manifiestas.

Aunque el análisis de clases latentes comparte ciertas características (los objetivos, por ejemplo) con otras técnicas clásicas de clasificación, como pueden ser el análisis de conglomerados jerárquico o el análisis de conglomerados de K-medias, existen particularidades que diferencian cada metodología específica. Una de las más importantes es que el análisis de clases latentes es un método de clasificación basado en el modelo. Esto quiere decir que la técnica propone un modelo estadístico para la población de la que se ha extraído la muestra de observaciones, a partir del cual se estiman los parámetros de las clases.

\subsection{Software estadístico}

Para llevar a cabo el análisis, se ha utilizado la versión 3.0.0 del software estadístico gratuito R. R es un programa modular en el que sus funcionalidades básicas pueden verse extendidas mediante la descarga e instalación de una gran variedad de paquetes 
adicionales que permiten la realización de multitud de análisis estadísticos. Entre ellos se encuentra mclust, el cual, según su propia definición, es un paquete para el ajuste mediante el algoritmo E-M de modelos de mezclas de variables normales para la clasificación de observaciones basada en el modelo. Será, por tanto, éste el paquete que se empleará en el análisis de los datos.

\section{RESULTADOS Y DISCUSIÓN}

Tras realizar un análisis descriptivo, se observa que la mayor parte de las medias de las variables oscilan en torno al valor 0 , siendo las variables cuya media es mayor que 0 aquellas que han experimentado una tasa de variación relativa media positiva en la totalidad de Andalucía. En el otro extremo, las variables con una media negativa son variables con una tasa de variación relativa media negativa en Andalucía. Por otro lado, se aprecia que la mayor parte de las variables presentan una varianza moderada, aunque también es cierto que un pequeño grupo (Plazas_Hoteles, Plazas_Hoteles_Apartamentos, Plazas_Apartamentos_1, Paro, Pantallas_Cine e Indice_Turistico) tienen varianzas muy grandes. Esto se debe a la presencia de observaciones extremas, con valores muy alejados con respecto a los del grueso de las observaciones.

Una vez comprobados los datos, y asegurado que dichas observaciones extremas no son fruto de ningún tipo de error, sino que son valores reales de las variables, se comienza con el análisis de clases latentes propiamente dicho. El primer paso es la determinación del número de grupos, $\kappa$, en los que se van a clasificar las observaciones. Se han calculado y comparado los valores del BIC para un total de diez modelos, considerando desde 1 a 9 grupos para cada modelo. Los resultados se muestran en la Tabla 2.

\section{Tabla 2}

\section{BIC PARA DIFERENTES MODELOS Y DIFERENTE NÚMERO DE GRUPOS}

\begin{tabular}{|lcccccccccc|}
\hline к & EII & VII & EEI & VEI & EVI & VVI & EEE & EEV & VEV & VVV \\
\hline $\mathbf{1}$ & 72976.24 & 72976.24 & 44886.87 & 44886.87 & 44886.87 & 44886.87 & 38543.44 & 38543.44 & 38543.44 & 38543.44 \\
\hline $\mathbf{2}$ & 68266.68 & 41069.18 & 43703.98 & 24043.30 & 31913.15 & - & 37873.27 & - & - & - \\
\hline $\mathbf{3}$ & 67725.61 & 36389.85 & 43001.58 & - & - & - & 37555.20 & 18341.53 & - & - \\
\hline $\mathbf{4}$ & 67312.48 & 34747.10 & - & - & - & - & 33605.35 & 21644.94 & - & - \\
\hline $\mathbf{5}$ & 67515.72 & 33539.52 & - & - & - & - & 36178.09 & 19362.05 & - & - \\
\hline $\mathbf{6}$ & 58324.25 & 32510.51 & - & - & - & - & 33347.87 & - & - & - \\
\hline $\mathbf{7}$ & 57431.79 & 31993.61 & 41232.43 & - & - & - & 33442.52 & - & - & - \\
\hline $\mathbf{8}$ & 57187.38 & 31355.81 & 40784.00 & - & - & - & 36002.98 & - & - & - \\
\hline $\mathbf{9}$ & 57280.85 & 30925.01 & 39213.30 & - & - & - & 33291.54 & - & - & - \\
\hline
\end{tabular}

Fuente: elaboración propia.

Estos resultados también se pueden representar gráficamente, para obtener una visión conjunta de los valores BIC para cada par modelo-número de componentes, como se puede observar en la Figura 4. 


\section{Figura 4 \\ EJEMPLO DE REPRESENTACIÓN GRÁFICA DEL BIC PARA DIFERENTES MODELOS Y DIFERENTE NÚMERO DE GRUPOS}

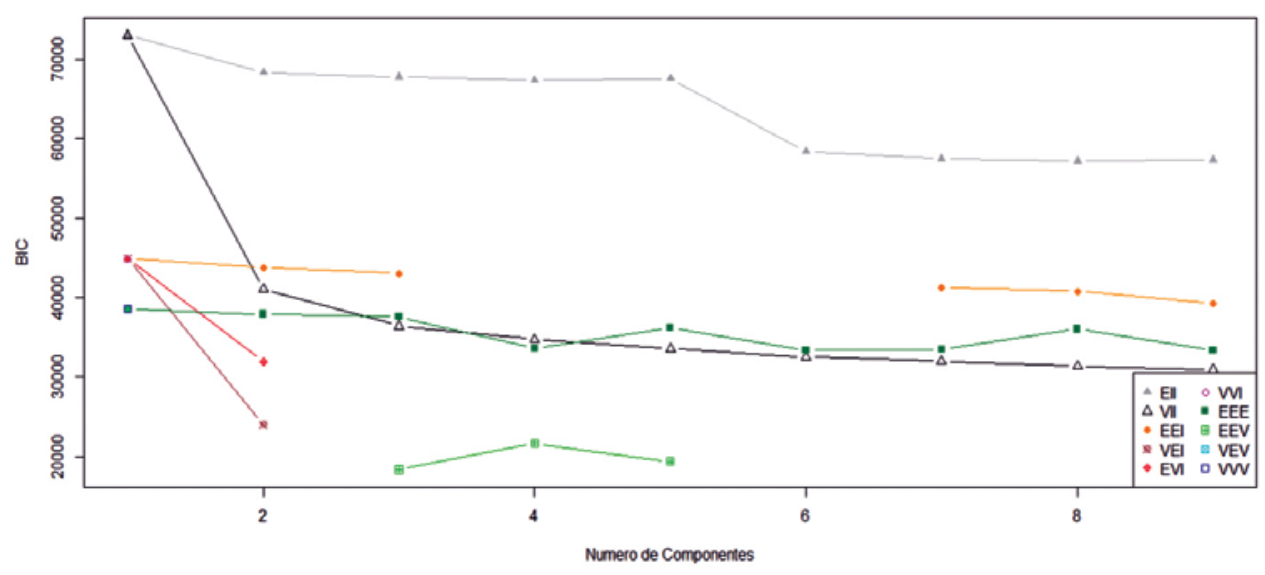

Fuente: elaboración propia.

Los diez modelos comparados se diferencian unos de otros en aspectos geométricos tales como la distribución, el volumen, la forma o la orientación de los grupos, aspectos que provienen, a su vez, de distintas consideraciones a la hora de la descomposición en valores propios de las matrices de varianzas-covarianzas de los distintos grupos, como se puede ver en la Tabla 3. La descomposición en valores propios de las matrices de varianzas-covarianzas de los grupos es la dada por:

$$
\Sigma_{k}=\lambda_{k} D_{k} A_{k} D_{k}^{T}
$$

siendo:

- $\lambda_{k}$ un escalar, que determina el volumen de los grupos.

- $D_{k}$ la matriz ortogonal que contiene los vectores propios de $\Sigma_{k}$ y que especifica la orientación de los grupos.

- $A_{k}$ una matriz diagonal cuyos elementos son proporcionales a los valores propios de $\Sigma_{k}$ y que determina la forma de los grupos, donde $B_{k}$ es una matriz diagonal tal que $\left|B_{k}\right|=1$. De este modo, los grupos son elípticos, pero paralelos a los ejes.

Atendiendo al criterio bayesiano, el modelo más adecuado sería el modelo elipsoidal, con igual forma, igual volumen y orientación variable de tres clases. Pero, antes de concluir la idoneidad de un modelo frente a los demás, es aconsejable calcular alguna otra medida adicional y contrastar los resultados. Por lo tanto, en la Tabla 4 se recogen los valores del ICL para los mismos diez modelos anteriores. 
Tabla 3

MODELOS PARA EL ANÁLISIS DE CLASES LATENTES

\begin{tabular}{|cccccc|}
\hline $\boldsymbol{\Sigma}_{\boldsymbol{k}}$ & Modelo & Distribución & Volumen & Forma & Orientación \\
\hline $\boldsymbol{\lambda} \boldsymbol{I}$ & EII & Esférica & Igual & Igual & - \\
\hline $\boldsymbol{\lambda}_{\boldsymbol{k}} \boldsymbol{I}$ & VII & Esférica & Variable & Igual & - \\
\hline $\boldsymbol{\lambda}_{\boldsymbol{B}}$ & EEI & Diagonal & Igual & Igual & - \\
\hline $\boldsymbol{\lambda}_{\boldsymbol{k}} \boldsymbol{B}$ & VEI & Diagonal & Variable & Igual & - \\
\hline $\boldsymbol{\lambda}_{\boldsymbol{B}}$ & EVI & Diagonal & Igual & Variable & - \\
\hline $\boldsymbol{\lambda}_{k} \boldsymbol{B}_{k}$ & VVI & Diagonal & Variable & Variable & - \\
\hline $\boldsymbol{\lambda}_{\boldsymbol{D} \boldsymbol{A} \boldsymbol{D}^{\boldsymbol{T}}}$ & EEE & Elipsoidal & Igual & Igual & Igual \\
\hline $\boldsymbol{\lambda}_{\boldsymbol{k}} \boldsymbol{A} \boldsymbol{D}_{\boldsymbol{k}}^{T}$ & EEV & Elipsoidal & Igual & Igual & Variable \\
\hline $\boldsymbol{\lambda}_{\boldsymbol{k}} \boldsymbol{D}_{\boldsymbol{k}} \boldsymbol{A} \boldsymbol{D}_{k}^{T}$ & VEV & Elipsoidal & Variable & Igual & Variable \\
\hline $\boldsymbol{\lambda}_{k} \boldsymbol{D}_{\boldsymbol{k}} \boldsymbol{A}_{\boldsymbol{k}} \boldsymbol{D}_{k}^{T}$ & VVV & Elipsoidal & Variable & Variable & Variable \\
\hline
\end{tabular}

Fuente: elaboración propia.

Tabla 4

ICL PARA DIFERENTES MODELOS Y DIFERENTE NÚMERO DE GRUPOS

\begin{tabular}{|lcccccccccc|}
\hline K & EII & VII & EEI & VEI & EVI & VVI & EEE & EEV & VEV & VVV \\
\hline $\mathbf{1}$ & 72976.24 & 72976.24 & 44886.87 & 44886.87 & 44886.87 & 44886.87 & 38543.44 & 38543.44 & 38543.44 & 38543.44 \\
\hline $\mathbf{2}$ & 68266.68 & 41070.68 & 43704.07 & 24043.30 & 31913.99 & - & 37873.27 & - & - & - \\
\hline $\mathbf{3}$ & 67726.72 & 36417.98 & 43007.70 & - & - & - & 37555.57 & $\mathbf{1 8 3 4 1 . 9 9}$ & - & - \\
\hline $\mathbf{4}$ & 67329.43 & 34800.97 & - & - & - & - & 33605.35 & 21645.02 & - & - \\
\hline $\mathbf{5}$ & 67698.54 & 33592.80 & - & - & - & - & 36230.34 & 19375.86 & - & - \\
\hline $\mathbf{6}$ & 58335.64 & 32568.15 & - & - & - & - & 33482.91 & - & - & - \\
\hline $\mathbf{7}$ & 57449.34 & 32043.72 & 41389.79 & - & - & - & 33610.60 & - & - & - \\
\hline $\mathbf{8}$ & 57207.57 & 31420.48 & 40978.15 & - & - & - & 36079.80 & - & - & - \\
\hline $\mathbf{9}$ & 57337.62 & 30990.51 & 39250.02 & - & - & - & 33449.48 & - & - & - \\
\hline
\end{tabular}

Fuente: elaboración propia.

Al igual que el BIC, el criterio ICL propone como mejor el modelo elipsoidal, con igual forma, igual volumen y orientación variable de tres clases, por lo que dicho modelo se designará como modelo final.

Una vez elegido el modelo, el siguiente paso consiste en la estimación de los parámetros, que recordemos son las medias y las matrices de varianzas-covarianzas para las variables dentro de cada grupo, así como los valores de la variable latente que indican la pertenencia de las observaciones a uno u otro grupo.

En las Tablas 5 y 6 se incluyen las estimaciones de la media y la varianza para cada variable dentro de cada grupo. Aunque las covarianzas en esas matrices no son exactamente 0 , la mayoría de ellas podría considerarse estadísticamente no significativas, dados los valores tan pequeños que presentan. 
Tabla 5

ESTIMACIÓN DE LA MEDIA PARA CADA VARIABLE DENTRO DE CADA GRUPO

\begin{tabular}{|c|c|c|c|}
\hline Variable & Grupo 1 & Grupo 2 & Grupo 3 \\
\hline Museos & 0.173060663 & 0.080999061 & 0.397845071 \\
\hline Plazas_Hoteles & 0.626153847 & 0.372980118 & 4.091914118 \\
\hline Plazas_Hoteles_Apartamentos & 0.368219325 & 0.000697975 & 3.089697013 \\
\hline Plazas_Hostales_Pensiones & 0.180196219 & 0.228060463 & 1.10962032 \\
\hline Plazas_Apartamentos_4 & 0.071625266 & 0 & 0.090936016 \\
\hline Plazas_Apartamentos_3 & 0.081165971 & 0.052715177 & 0.532758129 \\
\hline Plazas_Apartamentos_2 & 0.307955250 & 0.096782163 & 0.907268609 \\
\hline Plazas_Apartamentos_1 & 0.197977142 & 0.084377478 & 4.551795501 \\
\hline Desempleo & 1.199348638 & 0.948315079 & 1.058948521 \\
\hline Cines & -0.054293925 & -0.025105712 & -0.052751104 \\
\hline Pantallas_Cine & 0.017613815 & -0.028048364 & 2.160176255 \\
\hline Aforo_Cines & -0.003176231 & -0.023421738 & 0.408652802 \\
\hline Bancos & 0.410527008 & 0.54945873 & 0.822574575 \\
\hline Cajas_Ahorro & -0.211276733 & -0.395747413 & -0.031634417 \\
\hline Coop_Credito & 0.357800494 & 0.137267662 & 0.719792197 \\
\hline Campamentos & 0.065650320 & 0.041594487 & -0.058728874 \\
\hline Hoteles & 0.649904749 & 0.303967694 & 1.141982231 \\
\hline Hoteles_Apartamentos & 0.368859115 & 0.002278093 & 0.604399736 \\
\hline Hostales_Pensiones & 0.172510222 & 0.177870666 & 0.447475921 \\
\hline Oficinas_Turismo & 0.463434179 & 0.166055771 & 0.659284845 \\
\hline Restaurantes & 0.815756681 & 0.593245963 & 0.712769372 \\
\hline Cafeterias & 0.913765270 & 0.1104717 & 0.539016845 \\
\hline Taxis & 0.380435509 & 0.035543529 & 0.312152225 \\
\hline Coches_Alquiler_Conductor & 0.007958335 & 0.007565262 & -0.022192718 \\
\hline Ambulancias & 0.263742142 & -0.021408574 & 0.091737097 \\
\hline Transporte_Discrecional & 0.794492345 & 0.053729852 & 0.147081702 \\
\hline Transporte_Publico_Menos_10 & 0.379585841 & 0.028870226 & 0.696984269 \\
\hline Transporte_Publico_Mas_10 & 0.794492345 & 0.053729852 & 0.147081702 \\
\hline IAE_Division_6 & 0.464157300 & 0.412687579 & 0.606931958 \\
\hline Indice_Turistico & 0.046779614 & 0.022214513 & 1.739806664 \\
\hline
\end{tabular}

Fuente: elaboración propia. 


\section{Tabla 6}

ESTIMACIÓN DE LA VARIANZA PARA CADA VARIABLE DENTRO DE CADA GRUPO

\begin{tabular}{|c|c|c|c|}
\hline Variable & Grupo 1 & Grupo 2 & Grupo 3 \\
\hline Museos & 0.178111933 & 0.116975967 & 0.22004562 \\
\hline Plazas_Hoteles & 1.000186256 & 5.955465167 & 10.27848016 \\
\hline Plazas_Hoteles_Apartamentos & 0.262749547 & 0.015693157 & 6.458428094 \\
\hline Plazas_Hostales_Pensiones & 1.196425468 & 3.4189913 & 0.647958213 \\
\hline Plazas_Apartamentos_4 & 0.055921324 & 2.88923E-07 & 0.012114988 \\
\hline Plazas_Apartamentos_3 & 0.080634011 & 0.067569202 & 0.155634944 \\
\hline Plazas_Apartamentos_2 & 0.24136697 & 0.160068639 & 0.785894585 \\
\hline Plazas_Apartamentos_1 & 0.437959918 & 0.142299131 & 12.90398907 \\
\hline Desempleo & 1.627655497 & 13.0131463 & 0.09492286 \\
\hline Cines & 0.064560861 & 0.109812651 & 0.064717506 \\
\hline Pantallas_Cine & 0.142436919 & 0.098978921 & 1.829869336 \\
\hline Aforo_Cines & 0.095244319 & 0.110719493 & 0.238521983 \\
\hline Bancos & 0.23014103 & 0.839595954 & 0.225541655 \\
\hline Cajas_Ahorro & 0.226900204 & 0.510863856 & 0.043200133 \\
\hline Coop_Credito & 0.567942562 & 0.249515123 & 0.112712844 \\
\hline Campamentos & 0.205949783 & 0.12500125 & 0.027157423 \\
\hline Hoteles & 0.903887465 & 2.694173015 & 0.31676214 \\
\hline Hoteles_Apartamentos & 0.261854693 & 0.013738132 & 0.23518258 \\
\hline Hostales_Pensiones & 1.36624055 & 2.209282407 & 0.080236603 \\
\hline Oficinas_Turismo & 0.401372635 & 0.246641643 & 0.050270233 \\
\hline Restaurantes & 0.999956164 & 3.380943064 & 0.350666992 \\
\hline Cafeterias & 9.149112036 & 0.279389568 & 0.246183642 \\
\hline Taxis & 0.741274099 & 0.834009123 & 0.099284437 \\
\hline Coches_Alquiler_Conductor & 0.042137613 & 0.013898543 & 0.012533543 \\
\hline Ambulancias & 2.88423039 & 0.082085659 & 0.093688794 \\
\hline Transporte_Discrecional & 6.520749583 & 0.518089009 & 0.072120655 \\
\hline Transporte_Publico_Menos_10 & 1.068197394 & 0.884182037 & 0.725719766 \\
\hline Transporte_Publico_Mas_10 & 6.528167904 & 0.518089009 & 0.072120655 \\
\hline IAE_Division_6 & 0.181564226 & 0.555244287 & 0.014492316 \\
\hline Indice_Turistico & 0.339805132 & 0.838274595 & 1.534284715 \\
\hline
\end{tabular}

Fuente: elaboración propia. 
Una vez creados y caracterizados los grupos, es posible asignar cada una de las 769 observaciones a uno de ellos, cumpliendo así el objetivo que se planteó de segmentar el conjunto de datos.

A cada observación se le asigna una probabilidad de pertenencia a cada uno de los tres grupos creados, de manera que, finalmente, acaba asignándose a aquel de ellos en que dicha probabilidad es mayor.

En estos tres grupos segmentados, se pueden dividir los municipios en: destinos dinámicos, destinos altamente dinámicos y municipios en los que no se aprecia dinamismo (Figura 5). Seguidamente, se explican las características y la pertenencia de los municipios analizados a cada grupo.

\section{Figura 5 \\ SEGMENTACIÓN DE LOS MUNICIPIOS ANDALUCES EN FUNCIÓN DEL DINAMISMO ECONÓMICO DEL TURISMO (DET)}

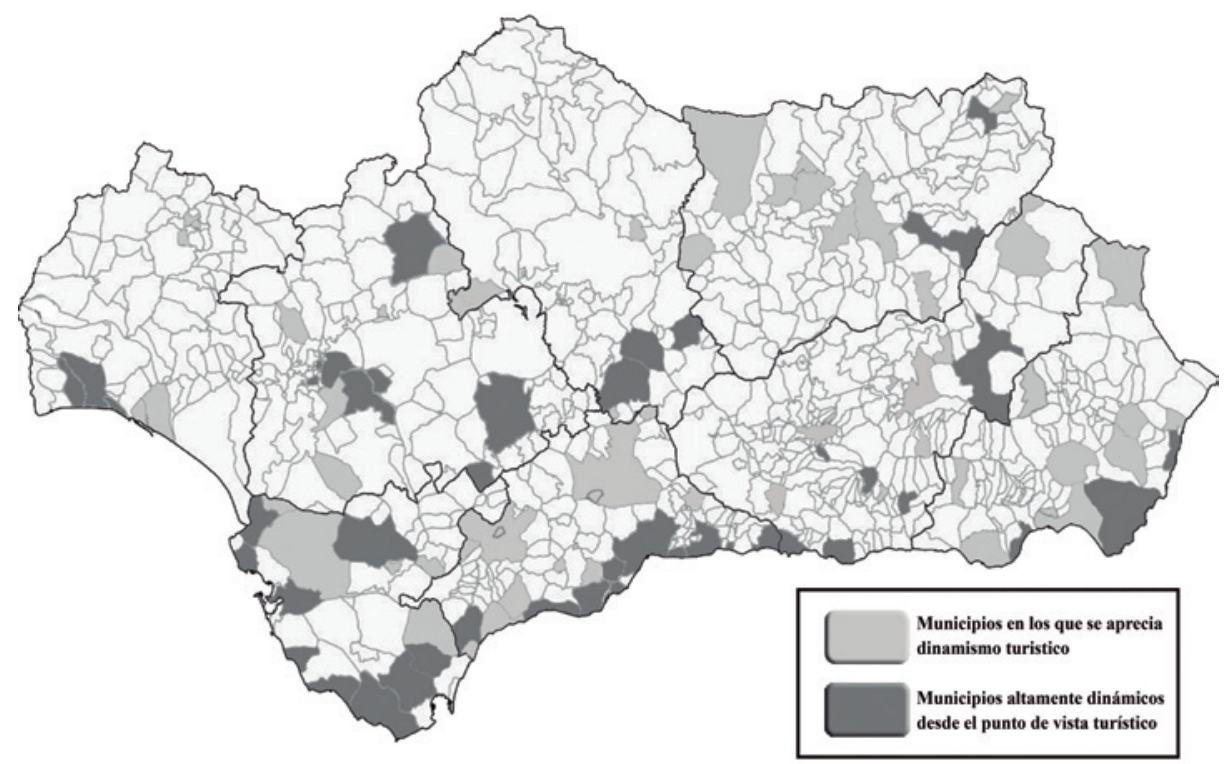

Fuente: elaboración propia.

\subsection{Destinos altamente dinámicos}

En la Tabla 7 se incluyen los 44 municipios que integran la clase latente que se ha denominado "destinos altamente dinámicos". Cabe destacar un primer grupo conformado por destinos turísticos tradicionales del litoral andaluz, como es el caso de Benalmádena, Fuengirola, Marbella, Mijas, Málaga o Nerja, en la Costa del Sol; Almuñecar y Motril, en la Costa Tropical de Granada; Barbate, Chiclana, Conil o Tarifa, en la costa gaditana; Mojácar, Níjar o Roquetas de Mar, en la costa almeriense, que cuentan con una amplia 
dotación no sólo de infraestructuras turísticas, sino también mejoras en la accesibilidad, en la oferta de bienes y servicios de ocio, etc., que justifican el alto dinamismo turístico experimentado por estos territorios durante el período analizado.

Tabla 7

DESTINOS ALTAMENTE DINÁMICOS

\begin{tabular}{|l|l|}
\hline Alcalá de Guadaíra & Mairena del Alcor \\
\hline Alhaurín de la Torre & Mairena del Aljarafe \\
\hline Almuñécar & Málaga \\
\hline Arcos de la Frontera & Marbella \\
\hline Barbate & Míjas \\
\hline Barrios, Los & Mojacar \\
\hline Baza & Motril \\
\hline Benalmádena & Nerja \\
\hline Cabra & Níjar \\
\hline Cádiar & Osuna \\
\hline Capileira & Pruna \\
\hline Cartaya & Puerta de Segura, La \\
\hline Casares & Punta Umbría \\
\hline Castellar de la Frontera & Rincón de la Victoria \\
\hline Chiclana de la Frontera & Roquetas de Mar \\
\hline Conil de la Frontera & Rota \\
\hline Constantina & Sanlúcar de Barrameda \\
\hline Fuengirola & Sevilla \\
\hline Iruela, La & Tarifa \\
\hline Lepe & Tomares \\
\hline Lucena & Vélez-Málaga \\
\hline Luque & Zubia, La \\
\hline
\end{tabular}

Fuente: elaboración propia.

El segundo grupo está formado por Sevilla y las ciudades que forman su área metropolitana (como Alcalá de Guadaira, Mairena del Alcor, Mairena del Aljarafe o Tomares), que han terminado conformando una oferta turística de ciudades dormitorio de una capital que tiene una indudable atracción turística. En un caso similar hay que considerar a ciudades dormitorio de Granada, como La Zubia.

En tercer lugar, es posible distinguir en este segmento un conjunto de ciudades de un tamaño poblacional mediano (20.000-40.000 habitantes), pero que cuentan con un rico patrimonio arquitectónico e histórico, al que, en los últimos años, han sumado una variada oferta cultural (museos, música, teatro, etc.) y de servicios turísticos (alojamiento, gastronomía, visitas turísticas, etc.) que les está permitiendo generar una atractiva oferta turística, que empieza a ser reconocida en los mercados, especialmente de turismo cultural, y muy apreciada por su calidad y singularidad. Estas ciudades se encuentran todavía en una fase muy 
temprana de desarrollo turístico, pero tienen un gran potencial para un futuro lleno de oportunidades en el segmento de turismo urbano-cultural (Pulido-Fernández y Sánchez-Rivero, 2010). Se trata de ciudades como Arcos de la Frontera, Baza, Cabra, Lucena u Osuna.

Un último grupo está formado por algunos municipios que, aprovechando la financiación europea destinada al fomento del desarrollo rural, el creciente interés de la demanda turística por la naturaleza y su pertenencia a alguno de los espacios naturales protegidos más emblemáticos de Andalucía, han generado una oferta turística rural y/o de naturaleza de enorme interés y dinamismo. Se trata, por ejemplo, de La Iruela o La Puerta de Segura, en el Parque Natural de Cazorla, Segura y Las Villas, o de Cádiar y Capileira, en La Alpujarra.

\subsection{Destinos dinámicos}

La Tabla 8 recoge los 56 municipios incluidos en la clase latente que se ha denominado "destinos dinámicos". Se trata de municipios cuyas probabilidades de pertenencia más elevadas y análisis gráfico permite considerar que disponen de un alto DET.

Un análisis más detallado de la Tabla 8 permite distinguir, a su vez, diferentes tipos de municipios y, con ello, diferentes comportamientos que explican el nivel que han alcanzado respecto al DET. Así, por ejemplo, cabe destacar que en este segmento existe un número importante de municipios de la provincia de Almería, una provincia en la que, durante el período estudiado, el turismo ha tenido un auge importante, auspiciado por la disponibilidad de recursos en un magnífico estado de conservación, la mejora de las infraestructuras de acceso, la inversión privada en una oferta de alojamiento de calidad media-alta y en atracciones vinculadas con los campos de golf o los parques de atracciones, así como el boom inmobiliario vinculado a segundas residencias generado con anterioridad a la crisis económica.

Otro grupo a destacar es el de las ciudades medias, como Antequera, Baeza, Guadix, Ronda o Úbeda, cuyas características más significativas ya fueron expuestas al analizar el segmento anterior.

Un tercer grupo a destacar en este segmento está conformado por un conjunto de municipios rurales que han sabido aprovechar las dinámicas generadas desde mitad de los noventa del siglo pasado al socaire de la financiación europea en apoyo del desarrollo rural. En este grupo hay que considerar municipios como Huéscar, Jimena de la Frontera, Torres de Albanchez, Ubrique o Vélez-Blanco.

En un cuatro grupo hay que considerar a las capitales de provincia (Almería, Cádiz y Granada) e, incluso, a Jerez de la Frontera y Linares. Se trata de ciudades con una elevada importancia poblacional, administrativa y comercial que, a su vez, tiene un efecto arrastre sobre el desarrollo turístico. El caso de Granada es, por supuesto, singular, al tratarse de uno de los principales destinos de turismo cultural de España.

Finalmente, también deben destacarse algunos municipios, como Benahavís, Estepona o Vera, cuya dinámica turística responde a una especialización de sol y playa, lo que, en principio, podría hacer pensar que deberían ser destinos turísticos altamente dinámicos, si bien, el comportamiento de algunas de las variables analizadas, especialmente el desempleo turístico, han limitado su dinamismo turístico. 
Tabla 8

DESTINOS DINÁMICOS

\begin{tabular}{|c|c|}
\hline Aldeire & Huescar \\
\hline Alfacar & Jayena \\
\hline Algar & Jerez de la Frontera \\
\hline Alhaurín el Grande & Jimena de la Frontera \\
\hline Almería & Laujar de Andarax \\
\hline Andújar & Linares \\
\hline Antequera & Manilva \\
\hline Arriate & Moguer \\
\hline Baeza & Montejaque \\
\hline Bailén & Palenciana \\
\hline Benahavís & Palma del Rio \\
\hline Burquístar & Palos de la Frontera \\
\hline Cabezas de San Juan, Las & Periana \\
\hline Cádiz & Porcuna \\
\hline Carpio, El & Puebla de los Infantes, La \\
\hline Cuevas de San Marcos & Ronda \\
\hline Dos Hermanas & Santa Ana La Real \\
\hline Ejido, El & Serón \\
\hline Estepona & Sorbas \\
\hline FuenteHeridos & Tabernas \\
\hline Galaroza & Tocina \\
\hline Gerena & Torres de Albanchez \\
\hline Granada & Trebujena \\
\hline Guadix & Úbeda \\
\hline Guaro & Ubrique \\
\hline Guarromán & Valle de Abdalajís \\
\hline Huércal de Almería & Velez-Blanco \\
\hline Huesa & Vera \\
\hline
\end{tabular}

Fuente: elaboración propia.

\subsection{Municipios no dinámicos}

Finalmente, hay una tercera clase latente, que se ha denominado "municipios no dinámicos". En este caso, no se habla exclusivamente de destinos, sino que se utiliza el término "municipios", ya que este segmento es muy heterogéneo, e incluye a municipios que sí que son destinos turísticos, pero también a otros muchos que en ningún caso pueden considerarse como destinos turísticos. Se trata de municipios cuyas probabilidades de 
pertenencia no son elevadas con respecto a las variables consideradas, o cuyos gráficos no aprecian un dinamismo aceptable. Dada la amplitud de este grupo, pues lo conforman 669 municipios, se ha optado por no incluir un listado de los mismos. Lógicamente, se trata de todos los municipios no incluidos en las Tablas 7 y 8 .

Respecto a este tercer grupo, cabe destacar la inclusión en él de capitales de provincia como Jaén, Córdoba o Huelva, o de tradicionales destinos turísticos, como Isla Cristina, Salobreña o Torremolinos. Es necesario, advertir que su inclusión en este grupo no significa que estos municipios no sean turísticos, sino que, durante el período analizado, no han experimentado variaciones significativas en ninguna de las variables que se han utilizado para medir el DET y, en consecuencia, la actividad turística de estas ciudades no se ha transformado sustancialmente, lo que implica falta de dinamismo. Esta circunstancia, por sí misma, ya está poniendo de manifiesto la necesidad de una intervención activa por parte de policymakers, gestores de estos destinos y sector privado, con el fin de generar una dinámica transformadora que convierta al turismo en un sector dinámico, capaz de contribuir a la mejora del desarrollo de estos territorios.

\section{CONCLUSIONES}

En este estudio se ha aplicado la técnica del análisis de clases latentes a un total de 769 observaciones (municipios) para las cuales se había anotado el valor de 30 tasas de variación relativa obtenidas a partir de la medición de otras tantas variables que miden el DET en dos instantes de tiempo. Como resultado del análisis, se han obtenido tres grupos de observaciones, los cuales han sido caracterizados a nivel estadístico y geométrico. Además, se ha dado una caracterización alternativa de los grupos en función del DET de los municipios que los conforman.

Los resultados obtenidos permiten validar la hipótesis establecida al inicio de esta investigación. A una escala local y con los indicadores de DET utilizados, se han podido segmentar tres grupos de municipios en función de su mayor o menor DET. En consecuencia, es posible conocer el carácter dinámico de cualquier destino y, a partir de ello, aplicar diferentes políticas turísticas en función de su nivel de dinamismo, así como conocer la contribución del turismo al desarrollo endógeno o local, para así demostrar que el turismo es una herramienta útil de desarrollo socioeconómico local y que, por tanto, en cualquier tipo de política pública deben de ir insertadas medidas para planificar y gestionar la actividad turística.

Este trabajo ha permitido una clasificación de los municipios andaluces, estableciéndose cuáles son las áreas comunes y a qué responden los territorios con un determinado nivel de DET y aquellos que no se clasifican dentro de dichos grupos.

Esta clasificación también ha permitido demostrar que los territorios que han sufrido una menor transformación en el período analizado no necesariamente son lugares en los que el turismo no es una actividad relevante, sino, más bien, lugares en los que la inversión y la capacidad de generación de empleo en el turismo ha sido muy limitada, de lo que se deduce que se necesita aplicar mejoras de gestión e inversiones para transformar esta actividad. 
Respecto a los municipios que son dinámicos o altamente dinámicos desde el punto de vista turístico, la influencia de factores como la ubicación de dichos territorios, la oferta de servicios turísticos y el empleo generado en esta actividad son las variables que, principalmente, han condicionado esta clasificación.

De acuerdo con la aproximación conceptual y la revisión de la literatura realizada en este artículo, es posible concluir las similitudes con la aproximación mesoeconómica del destino, donde las áreas de influencia turística suponen un gran apoyo y generación de valor en los territorios cercanos, alcanzando así las redes y relaciones mencionadas desde una perspectiva teórica. Es a partir de aquí cuando el concepto de distritos industriales y clusters en materia turística comienza a tener sentido, donde el propio dinamismo basado en el modelo del pentágono encuentra su aplicación, aunque de una manera parcial, con el desarrollo de la segmentación de municipios a través de su DET.

También, hay que indicar que, desde el punto de vista de los sistemas y las construcciones sociales, esta visión permite entender y analizar los destinos resultantes de las clasificaciones anteriores como entidades compuestas por personas e instituciones que desempeñan una determinada labor, dando valor añadido a los recursos ya existentes que otorgan un atractivo a un territorio. Por tanto, el componente humano, no sólo en la teoría, sino también en la práctica, debe de estudiarse y ponerse en valor.

Esta misma aproximación teórica y metodológica verifica la posible aplicación de políticas turísticas para planificar, gestionar y controlar un destino turístico, con la finalidad de hacerlo más sostenible, dotarlo de mejores infraestructuras, cumplir con los parámetros de gobernanza, generar un know-how y conocimiento tácito exclusivo en la gestión del destino que lo haga así único y destaque por su singularidad y, finalmente, se pueda desarrollar un entramado de servicios auxiliares que permitan un perfecto desarrollo de redes de cooperación y competencia entre empresas como condición indispensable del desarrollo turístico a escala mesoeconómica.

Por tanto, este análisis plantea una gran utilidad de cara a la diferenciación de políticas turísticas por grupos de municipios, lo que permite una mayor flexibilidad y un análisis más certero en la aplicación de dichas políticas.

Hay que tener en cuenta también que el escenario económico de crisis ha afectado a los resultados obtenidos, por lo que sería conveniente plantear el mismo análisis con los datos actualizados, una vez que las fuentes estadísticas permitan dicha actualización.

Como se ha explicado en el apartado metodológico, no ha sido posible medir todas las dimensiones que caracterizan el DT, al no existir información desagregada a nivel municipal de variables que conforman estas dimensiones. Por tanto, una de las propuestas a trabajar en el futuro, y que se extraen de este análisis, es la construcción de un sistema de indicadores que incluya todas las dimensiones que conforman el DT, lo que permitirá elaborar un análisis factorial que conduzca, a su vez, a un indicador sintético de DT.

Un siguiente paso y, por tanto, una línea de investigación futura, sería estudiar los segmentos identificados de una manera más detallada, lo que permitirá identificar los factores que favorecen o dificultan que un destino pueda ser dinámico desde el punto de vista turístico y que, en definitiva, explican la pertenencia de dichos territorios a los grupos que ha desvelado este análisis. 


\section{BIBLIOGRAFÍA}

AKAIKE, H. (1974): «A new look at the statistical model identification», IEEE Transactions on Automatic Control, $\mathrm{n}^{\circ} 19$ (6), pp. 716-723.

AURIOLES, J., FERNÁNDEZ, M.C. y MANZANERA, E. (2008): «El distrito turístico», Mediterráneo Económico, no 13, pp. 299-326.

BECATTINI, G. (1990): «The Marshallian Industrial District as a Socio-economic Notion» . En PYKE, F (Ed.): Industrial Districts and Inter-Firm Co-operation in Italy. Geneva, International Institute for Labour Studies, pp. 37-51.

BIERNACKY, C., CELEUX, G. y GOVAERT, G. (2000): «Assessing a mixture model for clustering with the integrated completed likelihood», IEEE T. Pattern. Anal., $\mathrm{n}^{\circ}$ 22 (7), pp. 719-725.

BLASCO, D., GUIA, J. y PRATS, L. (2014): «Tourism destination zoning in mountain regions: a consumer-based approach», Tourism Geographies, ${ }^{\circ}$ 16(3), pp. 512-528.

BODEGA, D., CIOCCARELLI, G. y DENICOLAI, S. (2004): «New Inte-Organizational Forms: Evolution of Relationship Structures in Mountain Tourism», Tourism Review, $\mathrm{n}^{\circ}$ 59(3), pp. 13-19.

CAMPRUBÍ, R., GUIA, J. y COMAS, J. (2008): «Destination Networks and Induced Tourism», Tourism Review, $\mathrm{n}^{\circ}$ 63(2), pp. 47-58.

CANDELA, G. y FIGINI, P. (2012): The Economics of Tourism Destinations. Berlin Heidelberg, Springer.

CAPONE, F. (2006): «Systemic Approaches for the Analysis of Tourism Destination: Towards the Tourist Local System». En LAZZERETTI, L. y PETRILLO, C. (Ed.): Tourism Local Systems and Networking. Amsterdam, Elsevier, pp. 7-23.

COCCOSSIS, H.; JANSSEN, H.; KIERS, M. y NIJKAMP, P. (1991): Tourism and strategic development, Serie Research Memoranda, Facultad de Economía y Econometría de la Universidad de Amsterdam.

CONSEJERÍA DE TURISMO, COMERCIO Y DEPORTE (2013): Balance de la actividad turística de Andalucía. Sevilla, Consejería de Turismo, Comercio y Deporte.

CUNHA BARBOSA, M. A. y ARICÓ ZAMBONI, R. (2001): «La formación de un Cluster en torno al turismo de naturaleza sustentable en Bonito, Brasil», Seminarios y Conferencias, $\mathrm{n}^{\circ} 17$, pp. 115-142.

DEMPSTER, A.P., LAIRD, N.M., y RUBIN, D.B. (1977): «Maximum Likelihood from Incomplete Data via the EM Algorithm», Journal of the Royal Statistical Society, ${ }^{\circ}$ 39(1), pp. 1-38.

DENICOLAI, S., CIOCARELLI, G. y ZUCCHELLA, A. (2010): «Resource-Based Local Development and Networked Core-competencias for Tourism Excellence», Tourism Management, $\mathrm{n}^{\mathrm{O}} 31(2)$, pp. 260-66.

FERNANDES, R. y THOMAS, W. (2012): «Factores humanos que influyen en el éxito o fracaso del turismo ambientalmente sustentable», Estudios y Perspectivas en Turismo, $\mathrm{n}^{\mathrm{o}} 21$, pp. 1433-1455.

FRANCH, M., MARTINI, U. y BUFFA, F. (2010): «Roles and opinions of Primary and Secondary Stakeholders within Community Type Destinations», Tourism Review, ${ }^{\circ}$ 65(4), pp. 74-86. 
HANNAN, E. J. y QUINN, B. G. (1979): «The Determination of the Order of an Autoregression», Journal of the Royal Statistical Society, n 41, pp. 190-195.

HAWKINS, D.E. (2004): «Sustainable Tourism Competitiveness Clusters: Application to World Heritage Sites Network Development in Indonesia», Asia Pacific Journal of Tourism Research, n $^{\circ}$ 9(3), pp. 293-307.

HJALAGER, A.M. (2000): «Tourism Destinations and the Concept of Industrial Districts», Tourism and Hospitality Research, $\mathrm{n}^{\circ}$ 2(3), pp. 99-112.

IWASHITA, C. (2003): «Media Construction of Britain as a Destination for Japanese Tourists: Social Constructionism and Tourism», Tourism and Hospitality Research, $\mathrm{n}^{\mathrm{o}}$ 4(4), p. 331.

LAZARSFELD, P.F. y HENRY, N.W. (1968): Latent Structure Analysis. Boston, Houghton Mifflin.

MERINERO-RODRÍGUEZ, R. y PULIDO-FERNÁNDEZ, J.I. (2009): «Desarrollo turístico y dinámica relacional. Metodología de análisis para la gestión activa de destinos turísticos», Cuadernos de Turismo, n 23, pp. 173-193.

PAVLOVICH, K. (2003): «The Evolution and Transformation of a Tourism Destination Network: The Waitomo Caves, New Zealand», L'Espace Géographique, n 10(3), pp. 201-213.

PEARCE, D.G. (2012): Frameworks for Tourism Research. Oxford, CABI.

PEARCE, D.G. (2014): «Toward an Integrative Conceptual Framework of Destinations», Journal of Travel Research, $\mathrm{n}^{\circ}$ 53, pp. 141-153.

PETRIC, L. y MRNJAVAC, Z. (2003): «Tourism Destination as a Locally Embedded System: Analogy between Tourism Destination and Industrial District», Tourism, $\mathrm{n}^{\mathrm{o}}$ 51(4), pp. 403-415.

PORTER, M.A. (1998): «Cluster and the new Economics of Competition», Harvard Business Review, November-December, pp. 77-90.

PULIDO-FERNÁNDEZ, J.I. y PULIDO-FERNÁNDEZ, M.C. (2013): Destinos turísticos. Conformación y modelos de gobernanza. En PULIDO-FERNÁNDEZ, J.I. y CÁRDENAS-GARCÍA, P.J. (Coord.): Estructura económica de los mercados turísticos. Madrid, Síntesis, pp. 179-204.

PULIDO-FERNÁNDEZ, J.I. y SÁNCHEZ-RIVERO, M. (2010): «Attitudes of the Cultural Tourist. A Latent Segmentation Approach», Journal of Cultural Economics, $\mathrm{n}^{\circ}$ 34(2), pp. 111-129.

RINGER, G. (1998): The Social Construction of Tourist Destination: The Process of Transformation of the Saariselka Tourism Region in Finnish Lapland. En RINGER, G. (ed.): Destination: Cultural Landscapes of Tourism. London, Routledge, pp. 154-173. SAARINEN, J. (2004): «Destinations in Change: The transformation Process of Tourist Destination», Tourist Studies, $\mathrm{n}^{\circ}$ 4(2), pp. 161-179.

SÁEZ, A. (2008): «El turismo rural como factor de desarrollo local». En PULIDOFERNÁNDEZ, J.I. (coord.): El turismo rural. Estructura económica y configuración territorial en España. Madrid, Editorial Síntesis, pp. 53-66.

SAINAGHI, R. (2006): «From Contents to Processes: Versus a Dynamic Destination Management Model (DDMM)», Tourism Management, $\mathrm{n}^{\circ}$ 27(5), pp. 1053-1063. 
SARANIEMI, S. y KYLÄNEN, M. (2011): «Problematizing the Concept of Tourism Destination: An Analysis of Different Theoretical Approaches», Journal of Travel Research, ${ }^{\circ}$ 50(2), pp. 133-143.

SCOTT, N., COOPER, C. y BAGGIO, R. (2008a): Network Analysis and Tourism: from Theory to Practice. Clevedon, Channel view.

SCOTT, N., COOPER, C. y R. BAGGIO, R. (2008b): «Destination Networks: Four Australian Cases», Annals of Tourism Research, $\mathrm{n}^{\circ} 35$ (1), pp. 169-86.

TIMUR, S. y GETZ, D. (2008): «A Network Perspective on Managing Stakeholders for Sustainable Urban Tourism», International Journal of Contemporary Hospitality Management, $\mathrm{n}^{\circ}$ 20(4), pp. 445-461.

VARISCO, C. (2004): «El cluster turístico de Miramar», Aportes y Transferencias, $\mathrm{n}^{\circ} 8$ (2), pp. 61-88.

WEAVER, D. y OPPERMANN, M. (2000): Tourism Management. Brisbane, Wiley. 
